\title{
Association of Hypoproteinemia in Preeclampsia with Maternal and Perinatal Outcomes : A Prospective Analysis of High-Risk Women
}

\section{Original Article}

\author{
Hossam El-Din Hussein Kamel, Adel Aly Elboghdady, Abdelsalam Mohamed \\ Abdelsalam Youssef
}

Department of Obstetrics and Gynecology, Faculty of Medicine, Al-Azhar University, Cairo, Egypt

\begin{abstract}
Background: Preeclampsia is a disorder of widespread vascular endothelial malfunction and vasospasm that occurs after 20 weeks gestation and can present as late as 4-6 weeks post-partum. It is clinically defined by hypertension and proteinuria, with or without pathologic edema.

Aim: The aim of the present study is to evaluate and assess the maternal and perinatal outcomes in preeclampsia (PE), according to serum albumin value.

Materials and Methods: In this study, 50 preeclamptic patients were divided into two groups according to the serum albumin level. To date, there has been no widely accepted predictive test or therapeutic intervention to prevent or delay preeclampsia. Patients included in the study were divided into two groups : group A preeclampsia with an albumin value of $>25 \mathrm{~g} / 1$ (mild hypoproteinemia). Group b preeclampsia with an albumin value of $<25 \mathrm{~g} / 1$ (severe hypoproteinemia). All the cases were subjected to full obstetric assessment and routine laboratory investigations including serum albumin value. Results: Severe hypoproteinemia has higher incidence of cesarean section delivery mode than mild hypoprteinemia. Severe hypopreteinemia has a higher percentage and risk of preterm labor than mild hypoprotenimia. Liver function and renal function tests are affected mainly with higher percentage in SHP than MHP. SHP is associated with more percentage of poor maternal and neonatal outcomes than MHP. Fetal growth restriction can be seen more often in SHP than mild hypoprotenimea.

Conclusion: Pre-eclampsia is a disease of multisystemic affection, causing liver and renal injuries, hematological abnormalities and abnormal uteroplacental blood flow. Identification of women at high risk for PE could potentially improve pregnancy outcome because intensive maternal and fetal monitoring in such patients would lead to an earlier diagnosis of the clinical signs of the disease and the associated fetal growth restriction and avoid the development of serious complications through such interventions as the administration of antihypertensive medication and early delivery. Estimation of albumin levels in pregnancy is of value in the early prediction of pre-eclampsia. SHP PE is associated with a higher risk of adverse maternal and neonatal outcomes than MHP PE, deserving closer surveillance during pregnancy..
\end{abstract}

Key Words: Hypoproteinemia, high risk women, preeclampsia

Received: 02 October 2019, Accepted: 24 October 2019

Corresponding Author: Abdelsalam Mohamed Abdelsalam Youssef, Department of Obstetrics and Gynecology, Faculty of Medicine, Al-Azhar University, Egypt, Tel.: 01003051610, E-mail: mohamedabdelsalam8778@gmail.com

ISSN: 2090-7265, August 2020, Vol.10, No. 3

\section{INTRODUCTION}

Preeclampsia (PE) is a leading cause of maternal and neonatal mortality and morbidity, complicating 3-5\% of all pregnancies ${ }^{[7]}$. Classically, the presence of proteinuria in women with gestational hypertension is clinically diagnosed as PE and is associated with increased maternal and neonatal morbidity and mortality compared with gestational hypertension $^{[11]}$

On a global scale, $\mathrm{PE}$ is responsible for approximately 50,000 maternal deaths annually ${ }^{[9]}$. PE frequently coexists with intrauterine growth restriction
(IUGR), placental abruption and sometimes the need of iatrogenic preterm delivery which are additional major causes of adverse outcomes ${ }^{[8]}$.

Risk factors for pre-eclampsia include obesity, prior hypertension, older age, and diabetes mellitus ${ }^{[1]}$. It is also more frequent in a woman's first pregnancy and if she is carrying twins. The underlying mechanism involves abnormal formation of blood vessels in the placenta amongst other factors. Most cases are diagnosed before delivery. Rarely, pre-eclampsia may begin in the period after delivery. While historically both high blood pressure and protein in the urine were required to make 
the diagnosis, some definitions also include those with hypertension and any associated organ dysfunction. Pre-eclampsia is routinely screened for during prenatal care $^{[10]}$.

PE develops after 20 weeks of gestation and is characterized by hypertension and proteinuria. Severe PE is often combined with hypoalbuminemia ; it was hypothesize that hypoalbuminemia may serve as an indicator of the severity of PE. Hypoalbuminemia occurs mainly due to systemic small vessel spasm, increased secretion of angiotensin and damaged and increased permeability of vascular endothelial cells, thereby leading to a large number of proteins and liquid leaking in tissue clearance and a loss of a large number of plasma proteins (especially serum albumin), causing intravascular dehydration ${ }^{[14]}$.

Although much research into mechanism of preeclampsia has taken place, its exact pathogenesis remains uncertain. Pre-eclampsia is thought to result from an abnormal placenta, the removal of which ends the disease in most cases $^{[1]}$.

During normal pregnancy, the placenta vascularizes to allow for the exchange of water, gases, and solutes, including nutrients and wastes, between maternal and fetal circulations. Abnormal development of the placenta leads to poor placental perfusion. The placenta of women with pre-eclampsia is abnormal and characterized by poor trophoblastic invasion ${ }^{[1]}$. It is thought that this results in oxidative stress, hypoxia, and the release of factors that promote endothelial dysfunction, inflammation, and other possible reactions ${ }^{[4]}$.

This intravascular dehydration may accelerate the appearance of intravascular lesions, which is thought to be a predisposing factor of the hemolysis, elevated liver enzymes and low platelet (HELLP) syndrome and acute fatty liver of pregnancy. In two recent studies, hypoproteinemia during pregnancy was observed in the PE group in the second trimester before the onset of clinical symptoms, and the authors suggest the possibility of preventing PE by maintaining adequate blood protein levels during early pregnancy ${ }^{[14]}$.

Complications of preeclampsia can affect both the mother and the fetus. Acutely, pre-eclampsia can be complicated by eclampsia, the development of HELLP syndrome, hemorrhagic or ischemic stroke, liver damage and dysfunction, acute kidney injury, and acute respiratory distress syndrome (ARDS) ${ }^{[2]}$. Preeclampsia is also associated with increased frequency of Caesarean section, preterm delivery, and placental abruption. Furthermore, an elevation in blood pressure can occur in some individuals in the first week postpartum attributable to volume expansion and fluid mobilization. Fetal complications include fetal growth restriction and potential fetal or perinatal death ${ }^{[12]}$.

Long-term, an individual with preeclampsia is at increased risk for recurrence of preeclampsia in subsequent pregnancies ${ }^{[6]}$.

The present study discussed the question ; Is hypoproteinemia effective at predicting perinatal risk in women with PE?

\section{AIM OF THE WORK}

The aim of the present study is to evaluate and assess the maternal and perinatal outcomes in preeclampsia (PE) according to serum albumin value.

\section{PATIENTS AND METHODS}

This prospective study was conducted at Sayed Galal Hopsital, Al-Azhar University between the time interval June till December 2017. The study included 50 preeclamptic pregnant women who were divided into 2 groups according to the serum albumin value. Group I PE with an albumin value of $>25 \mathrm{~g} / \mathrm{L}$ (mild hypoproteinemia [MHP]) and group II PE with an albumin value of $\leq 25 \mathrm{~g} / \mathrm{L}$ (severe hypoproteinemia [SHP]). For the study, SHP was defined as a serum albumin concentration of $\leq 25 \mathrm{~g} / \mathrm{L}$.

Patients were included in the study according to the following criteria : PE was defined according to the guidelines of the International Society for the Study of Hypertension in Pregnancy ; hypertension, blood pressure $\geq 14090 / \mathrm{mmHg}$ on at least two occasions and at least 4-6 h apart. The severe features such as severe hypertension, platelet count below $100,000 / \mu \mathrm{L}$, serum creatinine concentration of $1.1 \mathrm{mg} / \mathrm{dL}$ or doubling of the serum creatinine concentration in the absence of other renal disease, levels of liver transaminases elevated to twice the normal concentration, pulmonary edema, newonset cerebral or visual disturbance. Proteinuria ; more than $300 \mathrm{mg}$ protein in a $24-\mathrm{h}$ urine collection or a urine dipstick protein grade of $1+$ or greater.

While patients were excluded from the study according to the following criteria : kidney diseases, preexisting proteinuria, essential hypertension or other maternal chronic conditions (autoimmune diseases, diabetes, etc.) and twin pregnancies.

Maternal outcomes will be evaluated according to: delivery mode (spontaneous vaginal, instrumental vaginal, cesarean section $[\mathrm{CS}]$ ), eclampsia, HELLP syndrome, 
hypertension, abnormal liver function, abnormal renal function, ascites, oligohydramnios, thrombocytopenia and abruption placenta.

Neonatal outcomes will be evaluated according to perinatal deaths, preterm birth $(<37$ and 28 weeks gestation, spontaneous or iatrogenic) and fetal growth restriction (FGR, defined as actual birth weight below 10\% for gestational age). In the meantime, birth weight was also assessed.

All cases were informed by the study to declare their agreement to be in this study as agreed upon by the ethical committee.

\section{Data collection : 1- Complete history taking:}

Personal History: Name, age, race, parity, marital status, education, income, occupation, smoking history and plans for breastfeeding.

Obstetric history: Previous obstetric history, number of previous deliveries, delivery type and pregnancy complication as gestational hypertension, preeclampsia, gestational diabetes and antepartum or postpartum hemorrhage... etc

Past history: History of diabetes mellitus, hypertensive disorders, thyroid disease, chronic renal disease, liver disease, cardiac problems, previous operations and previous laparotomies.

Family history : History of gestational hypertension or preeclampsia.

2- Physical examination: including general blood pressure measurement, abdominal and pelvic examination.

3-Investigation: urine analysis, laboratory evaluation of blood hemoglobin, platelets, serum creatinine, serum uric acid, liver function test and serum albumin level.

\section{STATISTICAL ANALYSIS}

Data were collected, revised, coded and entered to the Statistical Package for Social Science (IBM SPSS) version 23 . The quantitative data were presented as mean standard deviations and ranges when their distribution found parametric. Also, qualitative variables were presented as number and percentages. The comparison between groups with qualitative data were done by using Chi-square test and Fisher exact test instead of the Chi-square only when the expected count in any cell found less than 5. The comparison between two groups with quantitative data and parametric distribution were done by using Independent t-test. The confidence interval was set to $95 \%$ and the margin of error accepted was set to $5 \%$.

\section{RESULTS}

In the present work, the mean maternal age was $34.66 \pm 4.52$ year in mild group versus $34.33 \pm 5.02$ year in sever group with non-statistical significant changes and the mean gestational age was $37.5 \pm 2.15$ week versus $35.5 \pm 1.98$ week with statistical significant differences. The analysis showed that women with severe hypoproteinemia were delivered almost 1.5 weeks earlier than that of mild hypoproteinemia group $(P=0.006)$ (Table 1$)$.

In the present study, there were $15(39.5 \%)$ cases in mild group versus $1(8.3 \%)$ case in sever group with vaginal delivery and the reminder cases with CS associated with $10(43.5 \%)$ with urgent CS versus $5(45.5 \%)$ with statistical significant differences and the reminder cases with CS with elective CS. In the SHP group, the percentage of CS was higher and the rate of spontaneous delivery was lower $(P=0.04$ and 0.04 respectively) (Table 2 ).

The mean highest systolic blood pressure during the course of disease was $152 \pm 12.5 \mathrm{mmHg}$ in mild cases versus $163 \pm 10 \mathrm{mmHg}$ in severe cases and diastolic blood pressure was $95 \pm 9.8 \mathrm{mmHg}$ versus $106 \pm 11.8 \mathrm{mmHg}$, respectively, with statistical significant differences. Severe hypertension was more frequent in SHP group than in MHP group ( $P=0.008$ and 0.002 respectively) (Table 3).

There were statistical significant decreased of abnormal liver and renal functions, ascites and abruption placenta in mild group when compared with severe group. In addition to insignificant decreased of eclampsia, HELLP syndrome, oligohydramnios and thrombocytopenia in mild cases versus sever group. Women with SHP were more likely to present with abnormal liver or/and renal function abnormality, ascitis and abruptio placenta than in MHP group (Table 4).

In the present study, there was significant decreased of poor maternal outcomes and poor neonatal outcomes in mild cases versus sever cases with statistical significant differences. SHP was found to be a highly significant risk factor for poor maternal outcomes $(P=0.005)$ and significant risk factor for neonatal outcomes $(P=0.040)$ (Table 5).

In this study, there was statistical significant increase of preterm birth in severe group when compared with mild cases associated with highly statistical increased of fetal growth restriction in severe cases. Neonatal outcomes were worse in the SHP group in comparison with MHP as it shows a highly significant difference in FGR $(P=<0.001)$ and a significant difference in preterm delivery $(P=0.035)$ (Table 6). 
Table 1: Maternal characteristics in mild hypoproteinemia and severe hypoproteinemia preeclampsia

\begin{tabular}{|c|c|c|c|c|c|c|}
\hline & & $\begin{array}{c}\text { Mild group } \\
\text { No. }=38\end{array}$ & $\begin{array}{c}\text { Severe group } \\
\text { No. }=12\end{array}$ & Test value & $P$-value & Sig. \\
\hline & Mean \pm SD & $34.66 \pm 4.52$ & $34.33 \pm 5.02$ & \multirow[b]{2}{*}{$0.211 \bullet$} & \multirow[b]{2}{*}{0.833} & \multirow[b]{2}{*}{ NS } \\
\hline Maternal age & Range & $27-43$ & $22-39$ & & & \\
\hline $\begin{array}{l}\text { Maternal age } \\
\text { groups }\end{array}$ & $\begin{array}{l}<=35 \text { years } \\
>35 \text { years }\end{array}$ & $\begin{array}{l}21(55.3 \%) \\
17(44.7 \%)\end{array}$ & $\begin{array}{l}6(50.0 \%) \\
6(50.0 \%)\end{array}$ & $0.102 *$ & 0.750 & NS \\
\hline $\begin{array}{l}\text { Gestational } \\
\text { age at delivery } \\
\text { (weeks ) }\end{array}$ & $\begin{array}{l}\text { Mean } \pm \text { SD } \\
\text { Range }\end{array}$ & $\begin{array}{c}37.5 \pm 2.15 \\
32-40\end{array}$ & $\begin{array}{c}35.5 \pm 1.98 \\
32-39\end{array}$ & $-2.859 \bullet$ & 0.006 & $\mathrm{~S}$ \\
\hline
\end{tabular}

NS: Non significant; S: Significant; HS: Highly significant

*:Chi-square test; $\bullet:$ Independent $\mathrm{t}$-test

Table 2: Comparison between mild hypoproteinemia and severe hypoproteinemia preeclampsia according to mode of delivery

\begin{tabular}{|c|c|c|c|c|c|c|}
\hline & & $\begin{array}{c}\text { Mild group } \\
\text { No. }=38\end{array}$ & $\begin{array}{c}\text { Severe group } \\
\text { No. }=12\end{array}$ & Test value & P-value & Sig. \\
\hline \multirow{3}{*}{ Vaginal delivery } & No & $23(60.5 \%)$ & $11(91.7 \%)$ & \multirow{3}{*}{4.064} & \multirow{3}{*}{0.043} & \multirow{3}{*}{$\mathrm{S}$} \\
\hline & & & & & & \\
\hline & Yes & $15(39.5 \%)$ & $1(8.3 \%)$ & & & \\
\hline \multirow{2}{*}{$\mathrm{CS}$} & No & $15(39.5 \%)$ & $1(8.3 \%)$ & \multirow{2}{*}{4.064} & \multirow{2}{*}{0.043} & \multirow{2}{*}{$\mathrm{S}$} \\
\hline & Yes & $23(60.5 \%)$ & $11(91.7 \%)$ & & & \\
\hline Urgent CS & & $10(43.5 \%)$ & $5(45.5 \%)$ & 4.166 & 0.041 & $\mathrm{~S}$ \\
\hline Elective CS & & $13(56.5 \%)$ & $6(54.5 \%)$ & 0.912 & 0.012 & S \\
\hline
\end{tabular}

NS: Non significant; S: Significant; HS: Highly significant

$*$ :Chi-square test; $\bullet:$ Independent t-test

Table 3: Comparison between highest systolic blood pressure and the highest diastolic blood pressure between mild and severe hypoproteinemia preeclampsia

\begin{tabular}{|c|c|c|c|c|c|c|}
\hline & & Mild group & Severe group & & & \\
\hline & & No. $=38$ & No. $=12$ & Test value* & P-value & Sig. \\
\hline $\begin{array}{l}\text { The highest systolic blood pressure } \\
\text { during the course of disease (mmh) }\end{array}$ & Mean \pm SD & $152 \pm 12.5$ & $163 \pm 10$ & 2.774 & 0.008 & $\mathrm{~S}$ \\
\hline $\begin{array}{l}\text { The highest diastolic blood pressure } \\
\text { during the course of disease (mmhg) }\end{array}$ & Mean \pm SD & $95 \pm 9.8$ & $106 \pm 11.8$ & 3.227 & 0.002 & $\mathrm{~S}$ \\
\hline
\end{tabular}

NS: Non significant; S: Significant; HS: Highly significant

*:Chi-square test 
Table 4: Shows laboratory investigations results and clinical data outcomes in both mild and severe hypoproteinemia preeclampsia

\begin{tabular}{|c|c|c|c|c|c|c|}
\hline & & $\begin{array}{c}\text { Mild group } \\
\text { No. }=38\end{array}$ & $\begin{array}{c}\text { Severe group } \\
\text { No. }=12\end{array}$ & Test value* & $P$-value & Sig. \\
\hline \multirow{2}{*}{ Eclampsia } & No & $37(97.4 \%)$ & $11(91.7 \%)$ & \multirow{2}{*}{0.772} & \multirow{2}{*}{0.380} & \multirow{2}{*}{ NS } \\
\hline & Yes & $1(2.6 \%)$ & $1(8.3 \%)$ & & & \\
\hline \multirow{2}{*}{ Hellp syndrome } & No & $35(92.1 \%)$ & $11(91.7 \%)$ & \multirow{2}{*}{0.002} & \multirow{2}{*}{0.961} & \multirow{2}{*}{ NS } \\
\hline & Yes & $3(7.9 \%)$ & $1(8.3 \%)$ & & & \\
\hline \multirow{2}{*}{$\begin{array}{l}\text { Abnormal liver } \\
\text { function }\end{array}$} & No & $34(89.5 \%)$ & $7(58.3 \%)$ & \multirow{2}{*}{5.992} & \multirow{2}{*}{0.014} & \multirow{2}{*}{$\mathrm{S}$} \\
\hline & Yes & $4(10.5 \%)$ & $5(41.7 \%)$ & & & \\
\hline \multirow{2}{*}{$\begin{array}{l}\text { Abnormal renal } \\
\text { function }\end{array}$} & No & $35(92.1 \%)$ & $8(66.7 \%)$ & \multirow{2}{*}{4.902} & \multirow{2}{*}{0.027} & \multirow{2}{*}{$\mathrm{S}$} \\
\hline & Yes & $3(7.9 \%)$ & $4(33.3 \%)$ & & & \\
\hline \multirow{2}{*}{ Ascites } & No & $34(89.5 \%)$ & $7(58.3 \%)$ & \multirow{2}{*}{5.992} & \multirow{2}{*}{0.014} & \multirow{2}{*}{$\mathrm{S}$} \\
\hline & Yes & $4(10.5 \%)$ & $5(41.7 \%)$ & & & \\
\hline \multirow{2}{*}{ Oligohydramnios } & No & $35(92.1 \%)$ & $9(75.0 \%)$ & \multirow{2}{*}{2.527} & \multirow{2}{*}{0.112} & \multirow{2}{*}{ NS } \\
\hline & Yes & $3(7.9 \%)$ & $3(25.0 \%)$ & & & \\
\hline \multirow{2}{*}{ Thrombocytopenia } & No & $35(92.1 \%)$ & $10(91.7 \%)$ & \multirow{2}{*}{0.780} & \multirow{2}{*}{0.377} & \multirow{2}{*}{ NS } \\
\hline & Yes & $3(7.9 \%)$ & $2(16.7 \%)$ & & & \\
\hline \multirow{2}{*}{ Abruption placenta } & No & $37(97.4 \%)$ & $9(75.0 \%)$ & \multirow{2}{*}{6.200} & \multirow{2}{*}{0.013} & \multirow{2}{*}{$\mathrm{S}$} \\
\hline & Yes & $1(2.6 \%)$ & $3(25.0 \%)$ & & & \\
\hline
\end{tabular}

NS: Non significant; S: Significant; HS: Highly significant

*:Chi-square test

Table 5: Association between severe hypoproteinemia preeclampsia with poor maternal and neonatal outcome in comparison with mild hypoproteinemia preeclampsia group

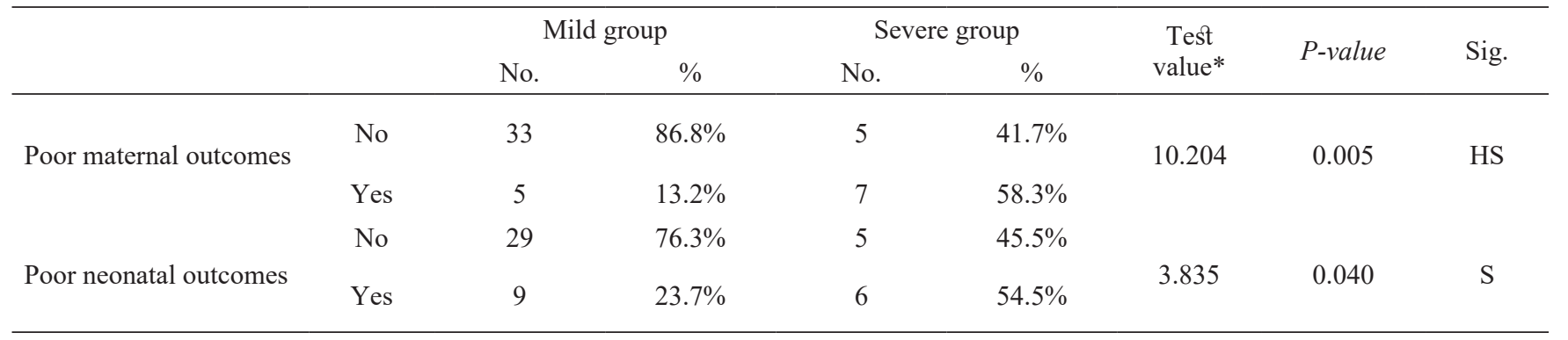

NS: Non significant; S: Significant; HS: Highly significant

*:Chi-square test

Table 6: Neonatal outcomes in mild hypoproteinemia and severe hypoproteinemia preeclampsia

\begin{tabular}{|c|c|c|c|c|c|c|c|c|}
\hline & & \multicolumn{2}{|c|}{ Mild group } & \multicolumn{2}{|c|}{ Severe group } & \multirow{2}{*}{$\begin{array}{c}\text { Test } \\
\text { value* }\end{array}$} & \multirow{2}{*}{ P-value } & \multirow{2}{*}{ Sig. } \\
\hline & & No. & $\%$ & No. & $\%$ & & & \\
\hline \multirow{3}{*}{ Perinatal death } & No & 38 & $100.0 \%$ & 11 & $91.7 \%$ & \multirow{3}{*}{3.231} & \multirow{3}{*}{0.072} & \multirow{3}{*}{ NS } \\
\hline & & & & & & & & \\
\hline & Yes & 0 & $0.0 \%$ & 1 & $8.3 \%$ & & & \\
\hline \multirow[b]{2}{*}{ Preterm birth } & No & 27 & $71.1 \%$ & 4 & $36.4 \%$ & \multirow[b]{2}{*}{4.417} & \multirow[b]{2}{*}{0.035} & \multirow[b]{2}{*}{ S } \\
\hline & Yes & 11 & $28.9 \%$ & 7 & $63.6 \%$ & & & \\
\hline \multirow[b]{2}{*}{ Fetal growth restriction } & No & 33 & $86.8 \%$ & 2 & $18.2 \%$ & \multirow[b]{2}{*}{19.706} & \multirow[b]{2}{*}{$<0.001$} & \multirow[b]{2}{*}{ HS } \\
\hline & Yes & 5 & $13.2 \%$ & 9 & $81.8 \%$ & & & \\
\hline
\end{tabular}

NS: Non significant; S: Significant; HS: Highly significant

*:Chi-square test 


\section{DISCUSSION}

In this study, we evaluate maternal and perinatal outcomes in 50 women with PE who were divided into two groups according to the different value of serum albumin $(\leq 25 \mathrm{~g} / \mathrm{L}$ and $>25 \mathrm{~g} / \mathrm{L})$.

In this study, there was no statistical significant difference between the two groups as regards the maternal age. Regarding the gestational age, this study showed that women with severe hypoalbuminemia were delivered almost 2 weeks earlier than those with mild hypoalbuminemia.

This is compatible with the study conducted in 2016 by $^{[5]}$ who reported that women with severe hypoalbuminemia were delivered almost 1.5 weeks earlier than the mild hypoalbuminemia group in which both studies shows significant difference in both groups regarding gestational age of delivery.

On the contrary ${ }^{[3]}$ showed in their study a highly significant difference $(P$ value $<0.001)$ in gestational age between both mild and severe hypoproteinemia conflicting with these results that represent a significant difference only.

In this study, severe hypoalbuminemia was a significant risk for CS $(P<0.05)$. Also, severe hypertension was more frequent with a significant value in the severe hypoalbuminemia group $(P 0.008)$. These results are similar to that reported by ${ }^{[5]}$.

In the current study, severe hypoalbuminemia was a significant risk factor for abnormal liver function, abnormal renal function, ascites and abruptio placenta $(P<0.05)$ for all. This again matches with the results reported by ${ }^{[5]}$ in 2016.

In contrast to these results ${ }^{[3]}$ study showed no significant difference between the two groups regarding abnormal renal function; while this study showed a significant difference $(P=0.027)$. Moreover, ${ }^{[3]}$ study showed no significant difference regarding aborptio placenta which again conflicts with these results.

Moreover, in ${ }^{[3]}$ study, there was a highly significant difference between the two groups regarding platelet concentration (thrombocytopenia) with $P$ value 0.001. However, in this study there was no significant difference regarding platelet concertation (thrombocytopenia) between the two groups.

Placental vascular spasm in PE leads to increased resistance to blood flow from the mother to the fetus resulting in chronic fetal hypoxia. In addition, $\mathrm{PE}$ is associated with loss of the mother's proteins. These factors can lead to intrauterine growth restriction and low birth weight ${ }^{[14,13]}$.

The results of this study supported this pathophysiology that women with severe hypoalbuminemia were more likely to deliver preterm infants $(P$ 0.035) and babies with fetal growth restriction $(P<0.001)$ than those with mild hypoalbuminemia.

Chen $^{[5]}$ in 2016 reported that preeclampsia with severe hypoalbuminemia was associated with significantly increased risk for preterm labor and low birth weight babies.

On the opposite, ${ }^{[3]}$ study showed no significant difference regarding FGR conflicting one more time with the results which showed a highly significant difference with a $P$ value $<0.001$.

The study showed that severe hypoalbuminemia is a risk factor for poor maternal outcome $(P$ 0.005) as well as poor neonatal outcome $(P$ 0.040). However, the main limitation of the study is the limited sample size. Further studies with enlarged samples should be performed to prove this data.

In this study, SHP was a significant risk factor for CS (91.7\% of cases) in comparison with (93.6 of cases) in HBC and XTW authors with a $P$ value in this study of 0.043 and in their study the $P$ value was 0.04 .

The study showed that severe hypoalbuminemia is a risk factor for poor maternal outcome $(P 0.005)$ as well as poor neonatal outcome $(P$ 0.040). However, the main limitation of the study is the limited sample size. Further studies with enlarged samples should be performed to prove this data. In this study, SHP was a significant risk factor for CS $(91.7 \%$ of cases) in comparison with (93.6 of cases) in HBC and XTW authors with a $P$ value in this study of 0.043 and in their study the $P$ value was 0.04 .

Hypoalbuminemia indicated that the conditions are further exacerbated in PE and further affects the function of various organs such as retinal damage, white matter lesions, pericardial effusion, ascites and liver and kidney dysfunction ${ }^{[15]}$.

In HBC and XTW analysis showed that there was a significant difference $\left(\begin{array}{ll}p & 0.04\end{array}\right)$ in gestational age of delivery between women with SHP and MHP; in which SHP delivered earlier than the MHP group by an average of 1.5-2 weeks. In this study, there was no significant difference in gestational age of delivery. 
In this study, there was no significant difference in spontaneous vaginal delivery between SHP and MHP which was not supported in HBC and XTW study in which there was significant difference between the two groups with a $P$ value of 0.04 .

In this study, the presence of SHP has been associated with increased risk of $\mathrm{CS}$, abnormal liver or renal function, ascites and thrombocytopenia and abruption placenta. In this study, the percentage of CS in SHP was $31.2 \%$ higher than in the MHP group; while in HBC and XTW study, the percentage in CS in SHP was $79 \%$ higher than MHP.

Placental vascular spasm in PE increases blood flow resistance from mother to the fetus resulting in chronic fetal hypoxia with a high number of the mother's protein lost and protein and other nutrient deficiencies, these factors can lead to intrauterine growth restriction and low birth weight ${ }^{[13,1]}$.

Neonatal outcomes were worse in the SHP group than mild hypoalbuminemia with a $p$ value 0.050 . Neonates of the women in the SHP group were more likely to have FGR which was highly significant in this study with a $p$ value 0.000 and significant in preterm deliveries with a $p$ value 0.035. HBC and XTW study supported these results with difference in $p$ value.

These results supported the pathophysiology described above where women with SHP were more likely to deliver preterm infants $34.7 \%$ more than MHP, FGR $68.6 \%$ than those with MHP PE.

Hypoalbuminemia is considered a factor of poor maternal and neonatal outcomes. In this study, SHP was a significant risk factor for CS (91.7\%), poor maternal outcomes $(25 \%)$ and poor neonatal outcomes (54.5) in PE women. The systemic small artery of women with PE was in spasm, protein absorption and utilization in the gastrointestinal tract were affected, often causing the emergence of hypoproteinemia. Hypoalbuminemia indicated that the conditions are further exacerbated in $\mathrm{PE}$ and further affects the function of various organs, such as retinal damage, white matter lesions, pericardial effusion, ascites and liver and kidney dysfunction ${ }^{[15]}$.

In this study, the presence of SHP has been associated with increased risk of CS, severe hypertension, abnormal liver or renal function, ascites and abruption placenta. The percentage of CS in SHP was $31.2 \%$, higher than in the MHP group. The incidence of abnormal liver or renal function in SHP $(41.7 \%$ and $33.3 \%)$ was significantly higher than in MHP $(10.5 \%$ and $7.9 \%$, respectively) and the difference was statistically significant $(P=0.014$ and 0.027 , respectively).

Placental vascular spasm in PE increases blood flow resistance from mother to the fetus resulting in chronic fetal hypoxia. With a high number of the mother's protein lost and protein and other nutrient deficiencies, these factors can lead to intrauterine growth restriction and low birth weight ${ }^{[14]}$. These results did not support that pathophysiology described above in which there wasn't significant difference between SHP and MHP in gestational age of delivery while supported the pathophysiology in regards of FGR as SHP showed $81.8 \%$ while MHP showed 13.2 with a $p$ value 0.000 .

It seems likely that many unknown risk factors may be present in women with PE and it is unclear whether this finding occurred as a pathophysiology of latent PE or was responsible for it. However, this study showed that SHP is a risk factor for women with PE. The main limitation of our study is the limited sample size. Further studies with enlarged samples should be performed to prove our data.

\section{CONCLUSION}

Pre-eclampsia is a disease of multisystemic affection causing liver and renal injuries, hematological abnormalities and abnormal uteroplacental blood flow. Identification of women at high risk for PE could potentially improve pregnancy outcome because intensive maternal and fetal monitoring in such patients would lead to an earlier diagnosis of the clinical signs of the disease and the associated fetal growth restriction and avoid the development of serious complications through such interventions as the administration of antihypertensive medication and early delivery. Estimation of albumin levels in pregnancy is of value in the early prediction of preeclampsia. SHP PE is associated with a higher risk of adverse maternal and neonatal outcomes than MHP $\mathrm{PE}$, deserving closer surveillance during pregnancy.

\section{CONFLICT OF INTEREST}

There are no conflicts of interests.

\section{REFERENCES}

1. Geneva:WHO.Worldhealth organization. Make every mother and child count. World health Report 2005. Hypertension in pregnancyReport of the American college of Obstetricians and gynecologists Task force on 
Hypertension in pregnancy. (PDF). Obstet Gynecol 2013; 122(5): 1122-31

2. Magee LA, Helewa M, Moutquin JM, et al. Diagnosis, evaluation, and management of the hypertensive disorders of pregnancy. Journal of Obstetrics \& Gynecology, Canada. 2008; 30:S148-

3. Hendrson JT,Whitlock EP, O'Connor, et al. A systemic evidence review for the US.preventive service Task force: Asprin for prevention of morbidity and mortality from preeclampsia. Annals of Internal Medicine 2014; 161(11):8119-826.

4. Hawkins TL, Roberts JM, Mangos GJ, Davis GK, Roberts LM, Brown MA. Plasma uric acid remains a marker of poor outcome in hypertensive pregnancy: A retrospective cohort study. BJOG. 2012;119:484-92.

5. Al-Jameil N, Aziz Khan F, Fareed Khan M, Q7 Tabassum H. A brief overview of preeclampsia. J Clin Med Res.2014;6:1-7.

6. Lambert $\mathrm{G}$, Brichant JF, Hartstein $\mathrm{G}$, et al. Preeclampsia: an update. Acta anaesthesiol Belg. 2014; 65(4):13749-.

7. Takahashi H, Hisano M, Sago H, Murashima A, Yamaguchi K. Hypoproteinemia in the second trimester among patients with preeclampsia prior to the onset of clinical symptoms. Hypertens Pregnancy. 2014;33:55-60.

8. Burton GJ, Yung HW, Cindrova-Davies T, et al. Placental endoplasmic reticulum stress and oxidative stress in the pathophysiology of unexplained intrauterine growth restriction and early onset preeclampsia. Placenta.2009; 30 (Suppl A): S438-.

9. Arulkumaran N and Lightstone L. Severe preeclampsia and hypertensive crises". Best Practice \& Research Clinical Obstetrics \& Gynaecology. 2013; 27 (6): 877-884.

10. Mustafa R, Ahmed S, Gupta A, Venuto RC. A Comprehensive Review of Hypertension in Pregnancy". Journal of Pregnancy 2012; 5: 1-19.

11. Cunningham F, Kenneth J, Steven LB et al.Hypertensive disorder in pregnancy. 2005;22:764.

12. Chen G, Wilson R, Wang U et al. Tumour necrosis factor-alpha (TNF-alpha) gene polymorphism and expression in pre-eclampsia. Clin Exp Immunol 1996; 104(1): 154-9.

13. Benoit J, Rey É (2011): Preeclampsia: should plasma albumin level be a criterion for severity? Journal of Obstetrics and Gynaecology Canada; 33(9):922-6.

14. Narasimha A, Vasudeva DS. Spectrum of changes in placenta in toxemia of pregnancy.Indian J Pathol Microbiol. 2011;54:15-20.

15. Tan A, Ma J, Deng X, Tang G. Severe ascites as the primary symptom of fulminant postpartum HELLP syndrome: A case report. Clin Exp Obstet Gynecol. 2015;42:685-7 